\title{
1 Electric Field-Assisted Orientation of Short Phosphate Glass Fibers 2 on Stainless Steel for Biomedical Applications
}

\author{
${ }_{3}$ Qiang Chen, ${ }^{\dagger \neq, \S \odot ~ J i a j i a ~ J i n g, ~}{ }^{\dagger}$ Hongfei Qi, ${ }^{\dagger}$ Ifty Ahmed, ${ }^{\|}$Haiou Yang, ${ }^{\ddagger}$ Xianhu Liu, ${ }^{\perp}$ T. L. Lu, ${ }^{* \dagger}$
} 4 and Aldo R. Boccaccini*, ${ }^{\infty}(0)$

$5{ }^{\dagger}$ Key Laboratory for Space Bioscience and Biotechnology, School of Life Sciences and ${ }^{\ddagger}$ State Key Laboratory of Solidification 6 Processing, Northwestern Polytechnical University, Xi'an 710072, Shaanxi, China

${ }_{7}^{8}$ Institute of Biomaterials, Department of Materials Science and Engineering, University of Erlangen-Nuremberg, Cauerstrasse 6,

8 Erlangen 91058, Germany

9 "Faculty of Engineering, Department of Mechanical, Materials and Manufacturing Engineering, University of Nottingham,

10 Nottingham NG 7 2RD, United Kingdom

$1{ }^{\perp}$ National Engineering Research Center for Advanced Polymer Processing Technology, Zhengzhou University, Zhengzhou 450002,

12 China

13 ABSTRACT: Structural and compositional modifications of metallic implant surfaces 14 are being actively investigated to achieve improved bone-to-implant bonding. In this 15 study, a strategy to modify bulk metallic surfaces by electrophoretic deposition (EPD) 16 of short phosphate glass fibers (sPGF) is presented. Random and aligned orientation of 17 sPGF embedded in a poly(acrylic acid) matrix is achieved by vertical and horizontal 18 EPD, respectively. The influence of EPD parameters on the degree of alignment is 19 investigated to pave the way for the fabrication of highly aligned sPGF structures in 20 large areas. Importantly, the oriented sPGF structure in the coating, owing to the 21 synergistic effects of bioactive composition and fiber orientation, plays an important role 22 in directional cell migration and enhanced proliferation. Moreover, gene expression of 23 MC3T3-E1 cells cultured with different concentrations of sPGF is thoroughly assessed 24 to elucidate the potential stimulating effect of sPGF on osteogenic differentiation. This 25 study represents an innovative exploitation of EPD to develop textured surfaces by 26 orientation of fibers in the macroscale, which shows great potential for directional 27 functionalization of metallic implants.

28 KEYWORDS: electrophoretic deposition, phosphate glass fiber, alignment, osteogenic differentiation, bone implants

\section{INTRODUCTION}

29 The long-term survival rate of metallic implants is usually 30 restricted due to their bioinert character, which leads to weak 31 bone-to-implant contact and eventually interfacial loosening in 32 situ. $^{1-3}$ The modification of implant surfaces with biological 33 coatings is being considered to improve osseointegration as 34 well as to reduce risks associated with infections and 35 corrosion. $^{4-6}$ Bioceramics, including bioactive glasses and 36 hydroxyapatite (HA), are well-known materials to functionalize 37 metallic implants. ${ }^{7,8}$ Inspired by the organic/inorganic 38 composition of natural bone, the combination of bioceramics 39 with biopolymers to form composite coatings is gaining 40 increasing interest. ${ }^{9}$ Such coatings are attractive because they 41 can be processed at room temperature and enable the 42 incorporation of biomolecules such as antibiotics during the 43 coating process. ${ }^{10}$ In this way, the bioceramic acts as the 44 bioactive filler and the biopolymer acts as a binder connecting 45 the filler particles to the metallic substrate. Compared to hard 46 coatings composed of pure ceramics, the presence of a 47 compliant polymer component is expected to promote close 48 contact between the metal implant and bone tissue. ${ }^{9}$



Phosphate glass fiber (PGF) possesses several attractive 49 features in comparison to those obtained from silicate bioactive 50 glasses, $^{11}$ e.g., PGF is completely resorbable in physiological 51 fluids, which is in contrast to the slow/incomplete degradation 52 of silica-based glasses. In addition, the fibrous structure of PGF 53 enables not only the reinforcing capability in composites but 54 also the biological functions in biomedical applications. Ahmed 55 et al. $^{12}$ produced an iron-doped PGF, which was seen to 56 support the attachment and differentiation of muscle precursor 57 cells along the fiber axis. Vitale-Brovarone et al. ${ }^{13}$ reported that 58 PGF played an important role on neuronal polarization and 59 further guided cell spreading. Other researches highlighted the 60 potential of PGF in promoting the functional recovery of 61 completely transected rat spinal cords ${ }^{14}$ and the peripheral 62 nerve injury ${ }^{15}$ in the way of controlling the directional cell 63 proliferation and differentiation. Indeed, many cell biology 64 studies have recently highlighted the importance of structural 65

Received: January 24, 2018

Accepted: March 5, 2018

Published: March 5, 2018 


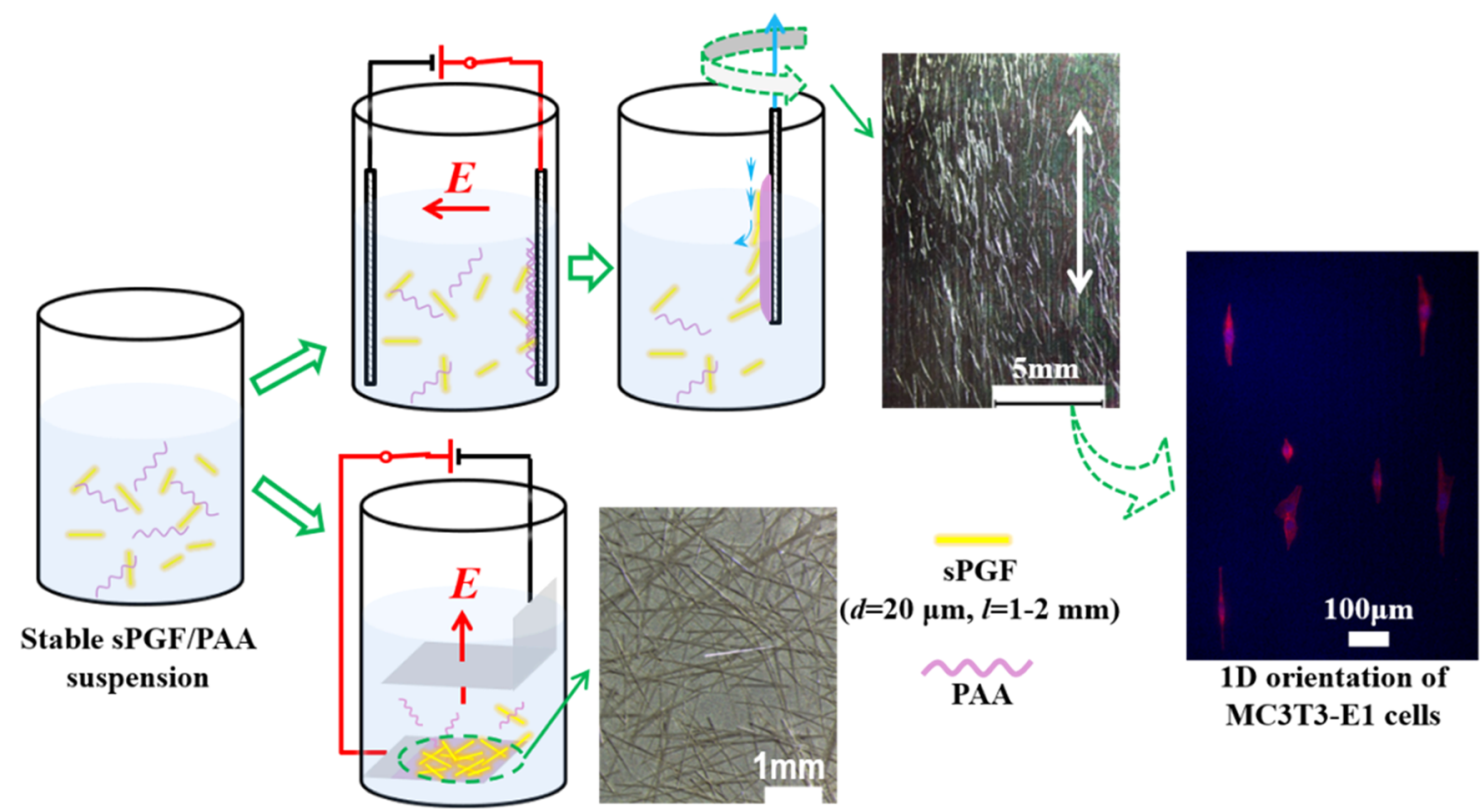

Figure 1. Vertical (top) and horizontal (bottom) EPD configurations for the production of aligned and random oriented sPGF on metallic substrates.

66 orientation on stimulating osteogenic behavior. ${ }^{16}$ It is 67 considered that random and aligned nanofibers will influence 68 the osteogenic gene expression by affecting the cell 69 morphology, e.g., randomly oriented fibrous scaffolds encour70 aged polygonal cell spreading and subsequent facilitate early 71 osteogenic differentiation, whereas aligned fibrous scaffolds 72 increased cell elongation and discouraged osteogenic differ73 entiation. ${ }^{17,18}$ However, Rowland et al. ${ }^{19}$ found that aligned 74 fibrous scaffolds using polydioxanone as raw material favored 75 significantly both osteogenic and chondrogenic differentiation 76 compared to random fibrous scaffolds. In addition, with the 77 incorporation of bioactive components, including nanohydrox78 yapatite and bone growth factors, Gao et al..$^{20}$ revealed that 79 aligned polycaprolactone nanofibers showed more osteogenic 80 activity than randomly oriented counterparts, a result that may 81 be assigned to a synergistic stimulation on the osteogenic 82 differentiation through an integrin-mediated pathway. In the 83 context of aligned fibrous coatings using PGF as building 84 blocks, it is expected that the presence of PGF, owing to its 85 bioactive character and fibrous geometry, will significantly 86 promote osteoblastic activity on the surface of bone implants. 87 Therefore, it is necessary to develop coating techniques that 88 enable incorporation of oriented PGF in novel coating 89 microstructures.

90 Indeed, numerous techniques have been developed to 91 produce aligned patterns or fibers aiming to explore their 92 effect on osteoblastic activity, such as electrospinning, ${ }^{21,22}$ 93 photolithography, ${ }^{23}$ anodization, ${ }^{24}$ and embossing techni94 ques. ${ }^{25}$ However, these techniques are mainly suitable to 95 produce aligned patterns on free-standing membranes or on flat 96 substrates in a relatively small size, which restricts their 97 potential application in orthopedic implants. There is a need for 98 straightforward, fast, and scalable patterning techniques, 99 including methods for self-assembly of micro/nanoparticles as 100 an alternative way to fabricate scalable patterns with controlled 101 nanostructures. ${ }^{26}$ In the case of short PGF (sPGF), the relative 102 small size and high flexibility of these fibers make them 103 promising for their assembly in aligned patterns on substrates with complex geometry and curvature. In addition, the 104 degradation rate, mechanical properties, and biological 105 functions can be easily changed by tailoring the ionic 106 composition of sPGF. ${ }^{27}$ To investigate the possible effects of 107 sPGF on regulating the osteoblastic proliferation and differ- 108 entiation, the prerequisite is to be able to manipulate the 109 orientation of SPGF in relative large areas and in controlled 110 manner.

Electrophoretic deposition (EPD) is a simple, rapid, and 112 versatile coating technique that is able to control the movement 113 of charged particles/molecules under an appropriate electric 114 field, leading to their consolidation on the oppositely charged 115 electrode with complex geometry and surface topography to 116 form coatings with tailored thickness and microstructure. ${ }^{28,29} 117$ According to our previous experience on EPD of oriented 118 cellulose nanowhisker films, ${ }^{30}$ the capability of EPD to control 119 the orientation of SPGF in an organic-inorganic composite 120 coating was investigated in this study. sPGF with the length of 121 1-2 $\mathrm{mm}$ were dispersed in aqueous poly(acrylic acid) (PAA) 122 solution to obtain stable EPD suspension at first. Both aligned 123 and random orientation of SPGF in the deposited coatings were 124 successfully obtained with the proper design of the EPD cell 125 and the corresponding EPD parameters, as schematically shown 126 in Figure 1. The mineralization of the deposited coatings as well $127 \mathrm{fl}$ as their interaction with osteoblasts (MC3T3-E1) in vitro was 128 investigated bearing in mind the potential application of the 129 coatings in bone replacement devices.

\section{EXPERIMENTAL SECTION}

2.1. Materials. Phosphate glass fibers with the composition 131 $40 \mathrm{P}_{2} \mathrm{O}_{5}-24 \mathrm{MgO}-16 \mathrm{CaO}-15 \mathrm{Na}_{2} \mathrm{O}-4 \mathrm{Fe}_{2} \mathrm{O}_{3}$ in mol \%-denoted as 132 $\mathrm{P} 40$ - were prepared as reported previously by Felfel et al. ${ }^{31}$ Briefly, 133 continuous fibers with a diameter of $\sim 15 \mu \mathrm{m}$ were first produced via 134 melt-draw spinning at $\sim 1100{ }^{\circ} \mathrm{C}$ and $\sim 1600 \mathrm{rpm}$, followed by 135 annealing at the glass transition temperature $\left(T_{\mathrm{g}}=479{ }^{\circ} \mathrm{C}\right)$ for $90 \mathrm{~min} .136$ The as-prepared fiber bundles were chopped into sPGF with the 137 length of 1-2 mm before use. Poly(acrylic acid) powder (PAA, CAS 138 no. 9001-03-4, and molecular weight $\sim 1250000 \mathrm{kDa}$ ) and other 139 
140 chemicals for simulated body fluid (SBF) preparation were purchased 141 from Sigma-Aldrich.

142 2.2. EPD-Based Coating Process. PAA was first dissolved in 143 deionized water by magnetic stirring for $10 \mathrm{~min}$ followed by $5 \mathrm{~min}$ 144 sonication treatment using an ultrasonic bath (Bandelin Sonorex, 145 Germany). The $\mathrm{pH}$ of the PAA solution was then adjusted to 8 by 146 adding a few drops of $\mathrm{NaOH}(1 \mathrm{M})$ to induce thorough ionization of 147 the PAA molecules. ${ }^{32}$ sPGF were then added in the PAA solution with 148 continuous magnetic stirring for $10 \mathrm{~min}$. Different concentrations of 149 PAA $(0.5-2 \mathrm{~g} / \mathrm{L})$ and sPGF $(0.25-2 \mathrm{~g} / \mathrm{L})$ were applied throughout 150 the experiment. Before each EPD process, the blend suspension was 151 put under continuous magnetic stirring to ensure the homogeneous 152 dispersion of sPGF. The EPD cell included two parallel 316L stainless 153 steel $\left(R_{\mathrm{a}}\right.$ of $\left.0.63 \mu \mathrm{m}\right)$ electrodes, horizontally or vertically located, with 154 the deposition area of $15 \times 20 \mathrm{~mm}^{2}$ and the distance of $10 \mathrm{~mm}$ 155 between electrodes. It should be noted that the deposition is also 156 feasible on other substrates with conductive surfaces. Anodic 157 deposition is achieved via the electrophoresis of negatively charged 158 PAA chains (due to ionization of $-\mathrm{COOH}$ to $-\mathrm{COO}-$ ) followed by 159 neutralization in the low $\mathrm{pH}$ region at the anode interface, as discussed 160 in the literature. ${ }^{33}$ According to a trial-and-error approach, processing 161 parameters were optimized to obtain composite sPGF-PAA coatings 162 with homogeneous and most importantly highly aligned sPGF (for 163 vertical electrode). The deposited anode was gently withdrawn from 164 the suspension $(\sim 2.5 \mathrm{~mm} / \mathrm{s})$ and dried horizontally in air for $30 \mathrm{~min}$ 165 at room temperature, yielding the deposit mass of sPGF-PAA 166 composite coatings in the range of $1-10 \mathrm{mg}$ (or $0.33-3.3 \mathrm{mg} / \mathrm{cm}^{2}$ ). 167 The alignment of sPGF in the coating and their robust adhesion to the 168 substrate were maintained due to the presence of a PAA binder layer. 169 The concentration of PAA and sPGF in the suspension, as well as the 170 corresponding EPD parameters intended to fabricate aligned sPGF by 171 horizontal electric field are listed in Table 1 . The alignment of sPGF in 172 the deposited sPGF-PAA coatings was characterized by optical 173 microscope (Leica, IC80HD).

Table 1. List of Suspensions and Corresponding EPD Parameters Using Horizontal Electric Field

\begin{tabular}{lcccc}
$\begin{array}{c}\text { sample } \\
\text { code }\end{array}$ & $\begin{array}{c}\text { PAA } \\
\text { concentration } \\
(\mathrm{g} / \mathrm{L})\end{array}$ & $\begin{array}{c}\text { sPGF } \\
\text { concentration } \\
(\mathrm{g} / \mathrm{L})\end{array}$ & $\begin{array}{c}\text { deposition } \\
\text { voltage }(\mathrm{V})\end{array}$ & $\begin{array}{c}\text { deposition } \\
\text { time }(\mathrm{s})\end{array}$ \\
I & 0.25 & 0.125 & 10 & 60 \\
II & 0.25 & 0.125 & 50 & 10 \\
III & 1 & 0.5 & 10 & 60 \\
IV & 1 & 0.5 & 20 & 30 \\
V & 1 & 0.5 & 30 & 15 \\
VI & 1 & 0.5 & 50 & 5 \\
VII & 2 & 0.5 & 20 & 15 \\
VIII & 2 & 1 & 20 & 15 \\
\hline
\end{tabular}

174 2.3. In vitro Mineralization Study. The as-prepared coatings 175 (e.g., sample I) were soaked in $20 \mathrm{~mL}$ of SBF prepared according to 176 the literature $e^{34}$ and placed in an orbital shaker at $37^{\circ} \mathrm{C}$ at an agitation 177 rate of $90 \mathrm{rpm}$. The samples were incubated for predetermined time 178 periods and the SBF was refreshed every 2 days. Then, the samples 179 were removed, gently rinsed with deionized water for three times and 180 dried at $37{ }^{\circ} \mathrm{C}$. Scanning electron microscopy (SEM) (model Auriga, 181 Zeiss) combined with energy-dispersive spectroscopy (X-MaxN 182 Oxford Instruments, U.K.) were applied to analyze the morphology (surface and cross section) and elemental composition of the samples 183 after mineralization. Ion release profiles of sPGF in SBF were studied 184 by means of inductively coupled plasma emission spectrometer 185 (ICPE9000, Shimadzu, Japan). sPGF (0.1 g) was soaked in $50 \mathrm{~mL}$ of 186 SBF and placed in an orbital shaker at $37^{\circ} \mathrm{C}$ with an agitation rate of 187 $90 \mathrm{rpm}$. After $2 \mathrm{~h}, 8 \mathrm{~h}, 1,6,14$, and 28 days, $1 \mathrm{~mL}$ of the solution was 188 extracted and filtered using $0.22 \mu \mathrm{m}$ filter to remove possible fiber 189 solids from the solution. The absorption wavelengths for the detection 190 of $\mathrm{P}$ and Ca were selected according to the literature. ${ }^{35}$ The calibration 191 curves were obtained by preparing standard solutions containing P and 192 Ca (supplied by Spex-CertiPrep, U.K.). Three replicates were 193 measured for each element at each time point.

2.4. Cellular Studies. 2.4.1. Cell Proliferation. MC3T3-E1 (pre- 195 osteoblast) cells were cultured in a growth medium of Alpha minimum 196 essential medium (Invitrogen) with 10\% fetal bovine serum, 100 unit/ 197 $\mathrm{mL}$ penicillin, and $100 \mathrm{mg} / \mathrm{mL}$ streptomycin. Cells were incubated at 198 $37{ }^{\circ} \mathrm{C}$ under a moist atmosphere of $5 \% \mathrm{CO}_{2}$ in air and the culture 199 medium was refreshed every 2 days. The cell proliferation was 200 determined using cell counting kit-8 assay (CCK-8; Dojindo, Japan). 201 The samples were sterilized by immersion in $70 \mathrm{vol} \%$ ethanol for 20202 min, followed by thorough rinsing with sterile phosphate-buffered 203 saline (PBS) before use. Pure PAA coatings produced from sPGF-free 204 suspension were chosen as the control to the sPGF-PAA coatings 205 (sample VI in this study). $5 \times 10^{4}$ cells in $100 \mu \mathrm{L}$ medium were first 206 seeded on the sterilized samples $\left(1 \times 1 \mathrm{~cm}^{2}\right)$ located in a 24-well plate. 207 After $4 \mathrm{~h}$ of incubation to ensure complete adherence of the cells on 208 the sample, each well was supplemented with $900 \mu \mathrm{L}$ medium. In this 209 way, all the cells were seeded only on the sample rather than on the 210 uncovered well plate. After culturing for 2, 4, and 7 days, $20 \mu \mathrm{L}$ per 211 well of CCK-8 reagent was added and the cells were incubated for 212 additional $2 \mathrm{~h}$. The results were expressed as the mean absorbance 213 (optical density at $450 \mathrm{~nm}$ ) of six parallel tests.

2.4.2. Cell Morphology. For cell morphology observations, the cells 215 were incubated on both the control and the sPGF-PAA samples for 2216 days following the above-mentioned protocol. The cytoskeleton of 217 MC3T3-E1 was analyzed using filamentous actin (F-actin) staining. 218 Briefly, the cells were fixed with 4\% paraformaldehyde for 10-30 min, 219 permeabilized with $0.1 \%$ Triton X-100 for $5 \mathrm{~min}$, and blocked with $1 \% 220$ bovine serum albumin for $20 \mathrm{~min}$. The treated skeleton and nuclei 221 were then stained with rhodamine phalloidin (100 nM, Solarbio, 222 China) for $20 \mathrm{~min}$ and Hoechst $33258(10 \mu \mathrm{g} / \mathrm{mL}$, Solarbio, China) 223 for $5 \mathrm{~min}$ in the dark, respectively. The stained cells were rinsed 224 thoroughly with PBS and then viewed under fluorescence microscopy 225 (Nikon 80i, Japan). High-magnification images of cell morphology on 226 the sPGF-PAA coating were obtained by SEM (model Auriga, Zeiss). 227 The samples were rinsed with PBS and fixed with $2.5 \%$ glutaraldehyde 228 for $3 \mathrm{~h}$ at $4{ }^{\circ} \mathrm{C}$, and subsequently dehydrated in ethanol solutions of 229 varying concentrations $(30,50,70,90$, and $100 \%$ ) for $15 \mathrm{~min}, 230$ respectively. The samples were dried in a desiccator at $37^{\circ} \mathrm{C}$ and then 231 sputter-coated with gold before SEM observation.

2.4.3. Osteogenic Differentiation Effect of SPGF. The osteogenic 233 markers, including cell morphology, and bone-related gene expression 234 were characterized by culturing MC3T3-E1 cells with different 235 concentrations of $\operatorname{sPGF}(0,1,5 \mathrm{mg} / \mathrm{mL})$. The expression of genes 236 associated with osteogenesis, including runt-related transcription factor 237 2 (RUNX2), collagen type I (COL1 $\alpha$ ), and osteocalcin (OCN), was 238 assessed by quantitative real-time polymerase chain reaction (qRT- 239 PCR). Briefly, $2.5 \times 10^{5}$ cells per well were seeded in six-well plate 240 with SPGF and cultured for 7 and 14 days. During culture, the 241 morphology of the cells was observed by optical microscope (CKX41, 242

Table 2. Primer Sequences of Genes for the Quantitative RT-PCR

gene name (genebank no.)

RUNX2 (NM_001145920.1)

COLI $\alpha$ (NM_007742.3)

OCN (NM 007541.2)

GAPDH (NM 008084.2) forward primer sequence $\left(5^{\prime}-3^{\prime}\right)$

TGCACCTACCAGCCTCACCATAC

GAAGGCAACAGTCGATTCACC

AGGACCATCTTTCTGCTCACT

TGCACCACCAACTGCTTAG reverse primer sequence $\left(5^{\prime}-3^{\prime}\right)$

GACAGCGACTTCATTCGACTTCC

GACTGTCTTGCCCCAAGTTCC

GCGTTTGTAGGCGGTCTTCA

GGATGCAGGGATGATGTTC 

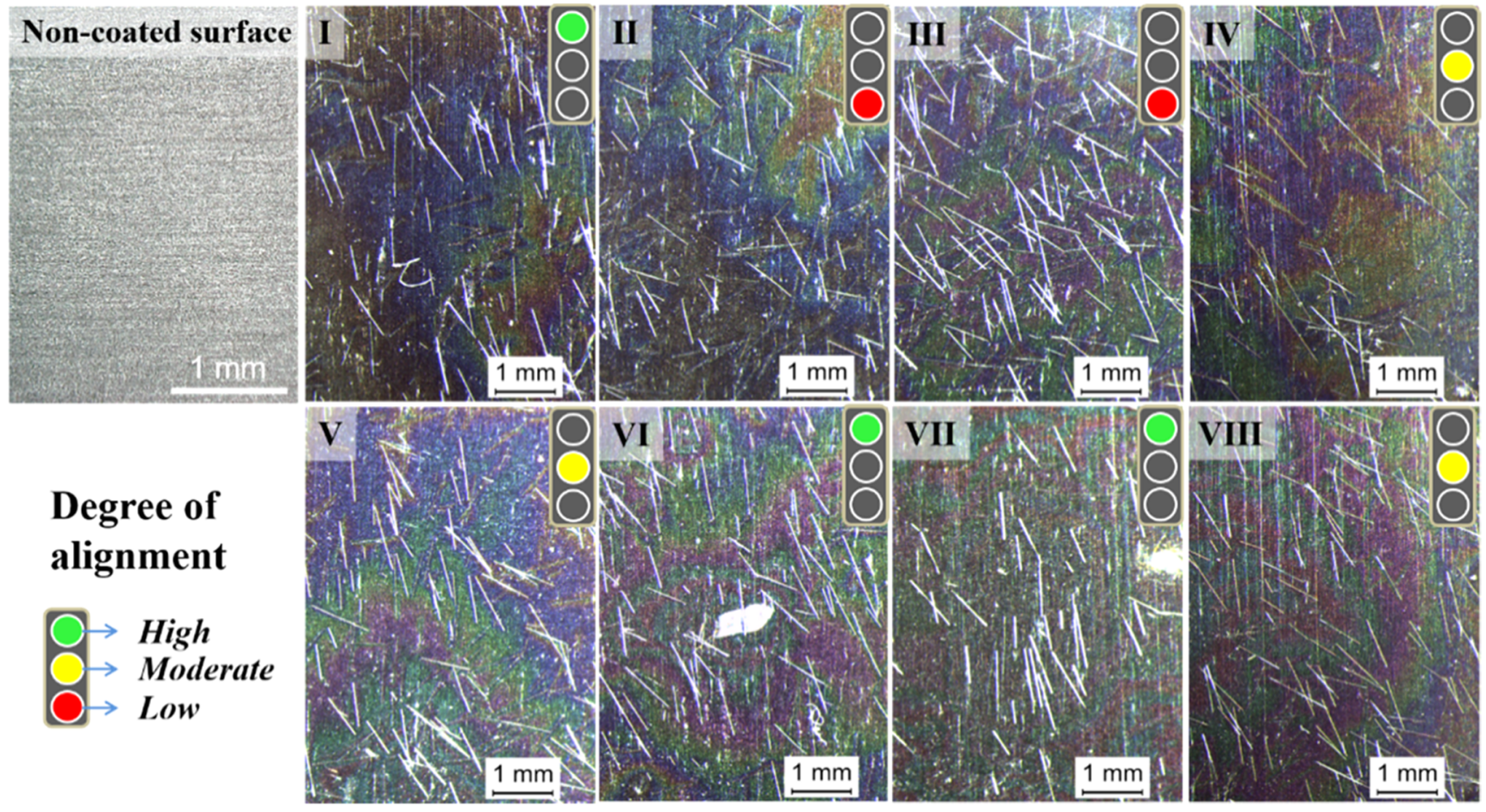

Figure 2. Alignment of sPGF in the deposited coatings ((I)-(VIII), compared to noncoated surface) prepared using different EPD parameters under horizontal electric field. The degree of alignment was assigned to three levels (high, moderate, and low levels) indicated by green, yellow, and red symbols, respectively.

243 OLYMPUS). After culture, the total RNA was extracted using TRIzol 244 reagent (TaKARA Bio Inc, Shiga, Japan) and then converted into 245 cDNA using PrimeScript RT Master Mix (TaKARA Bio Inc, Shiga, 246 Japan) according to the manufacturer's instructions. The gene 247 expression analysis was performed using CFX Manager System 248 (CFX96TM, BioRad) and SYBR Premix Ex Taq II (TaKARA Bio 249 Inc, Shiga, Japan) $(n=3)$. The relative transcript quantities of genes 250 were normalized using GAPDH as the endogeneous reference gene. 251 The primer sequences of the genes in this study are listed in Table 2 252 according to the literature.

253 2.4.4. Alkaline Phosphatase (ALP) Activity Test. The ALP activity 254 of cells cultured with different concentrations of sPGF was tested using 255 an ALP assay kit (Beyondtime Bio-Tech, China). It should be noted 256 that osteogenic medium consisting of normal culture medium 257 supplemented with $0.01 \mathrm{M} \beta$-glycerophosphate and $50 \mu \mathrm{g} / \mathrm{mL}$ vitamin $258 \mathrm{C}$ was used for this experiment. $1 \times 10^{5}$ cells per well were seeded with 259 different concentrations of $\mathrm{sPGF}(0,1,5 \mathrm{mg} / \mathrm{mL})$ and cultured for 7 , 26014 , and 21 days. After culture, the samples were rinsed with PBS and 261 lysed with $0.1 \%$ Triton X-100 solution for $30 \mathrm{~min}$ at $4{ }^{\circ} \mathrm{C}$. Then, the 262 solution was centrifuged at $3000 \mathrm{rpm}$ for $2 \mathrm{~min}$ at $4{ }^{\circ} \mathrm{C}$. $50 \mu \mathrm{L}$ of the 263 obtained supernatant was mixed with $50 \mu \mathrm{L}$ chromogenic substrate 264 and cultured for $30 \mathrm{~min}$ at $37^{\circ} \mathrm{C}$. The reaction was stopped by adding $265100 \mu \mathrm{L}$ terminal liquid. The ALP activity was measured in 266 quadruplicate at $405 \mathrm{~nm}$ using a microplate reader.

267 2.5. Statistical Analysis. The results in this study were expressed 268 as mean \pm standard deviation (SD) from at least three independent 269 experiments. The statistical analyses between the analyzed groups were 270 carried out by SPSS 16.0 software based on the one-way analysis of 271 variance. Statistical significance was considered when $P<0.05,0.01$, 272 and 0.001 .

\section{RESULTS AND DISCUSSION}

\section{3.1. Orientation of sPGF with Vertical and Horizontal} 274 Electric Field. PAA is known as a biodegradable and 275 biocompatible polymer, which is promising for the assembly 276 of biomedical devices. ${ }^{37,38}$ The role of PAA during the 277 processing of sPGF-PAA composites can be assigned to three aspects. First, the high viscosity of PAA solution at pH 8278 facilitated a stable dispersion of sPGF in the suspension. In 279 addition, according to the findings on electrophoretic co- 280 deposition of $\mathrm{PAA}-\mathrm{TiO}_{2}$ composite coatings, ${ }^{39}$ it is assumed in 281 the present experiment that SPGF were wrapped with PAA 282 molecules leading to their negative surface charge and their 283 further deposition on the positive electrode under the electric 284 field. Third, the simultaneous deposition of PAA could act as 285 the binder connecting sPGF to the surface of the substrate. As 286 schematically shown in Figure 1, a vertical electric field was first 287 designed to produce random orientation of sPGF in the sPGF- 288 PAA coatings. Due to the effect of gravity and electrophoresis 289 of PAA-charged sPGF, an accelerated sedimentation of SPGF 290 could be achieved. On the other hand, horizontal electric field 291 was applied with the aim to achieve one-dimensional 292 orientation of SPGF in the deposited coating. During the 293 withdrawal process of the coated electrode, the suspended as 294 well as the weakly adhered SPGF were aligned on the PAA gel 295 along the withdrawal direction, leading to a vertical orientation 296 of sPGF in the coating.

297

Various suspension compositions and EPD parameters 298 (Table 1) were used to achieve highly aligned sPGF in the 299 deposited coating. The optical microscopic images of sPGF in 300 the coating as well as the noncoated surfaces are shown in 301 Figure 2. The degree of alignment was assigned to three levels, $302 \mathrm{f} 2$ which are indicated by green, yellow, and red symbols, 303 respectively. According to this visual inspection of the 304 microscopic images, it was found that the deposition time 305 played a negative effect on the degree of alignment as the 306 amount of randomly deposited sPGF increased with the 307 deposition time. The effect of deposition voltage on the degree 308 of alignment was investigated when comparing the orientation 309 of sPGF on samples I and II, which indicated a higher degree of 310 sPGF orientation obtained at lower voltages. However, 311 

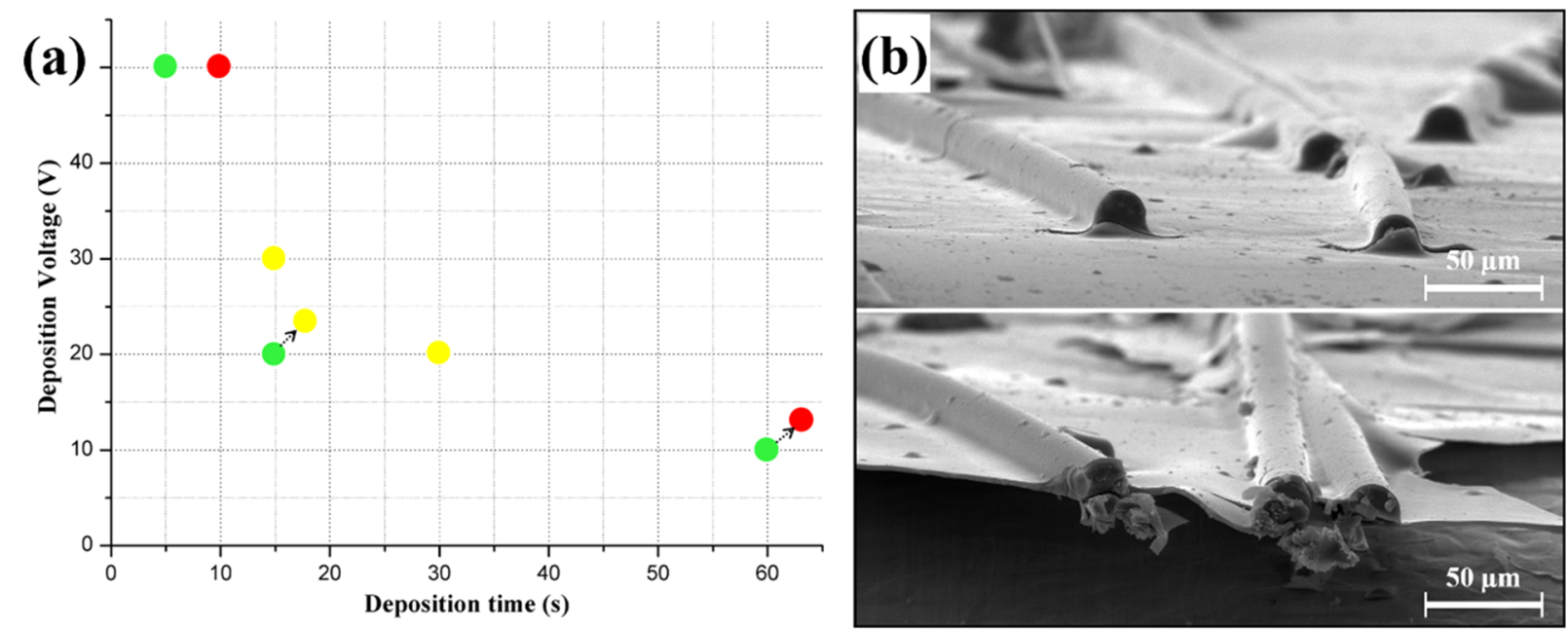

Figure 3. (a) Degree of sPGF alignment distributed in a deposition voltage vs deposition time plot. The arrows indicated the reduction of sPGF alignment with increasing sPGF concentration. (b) Cross-sectional SEM images of sample VI indicating that sPGF were partially embedded in the PAA layer.
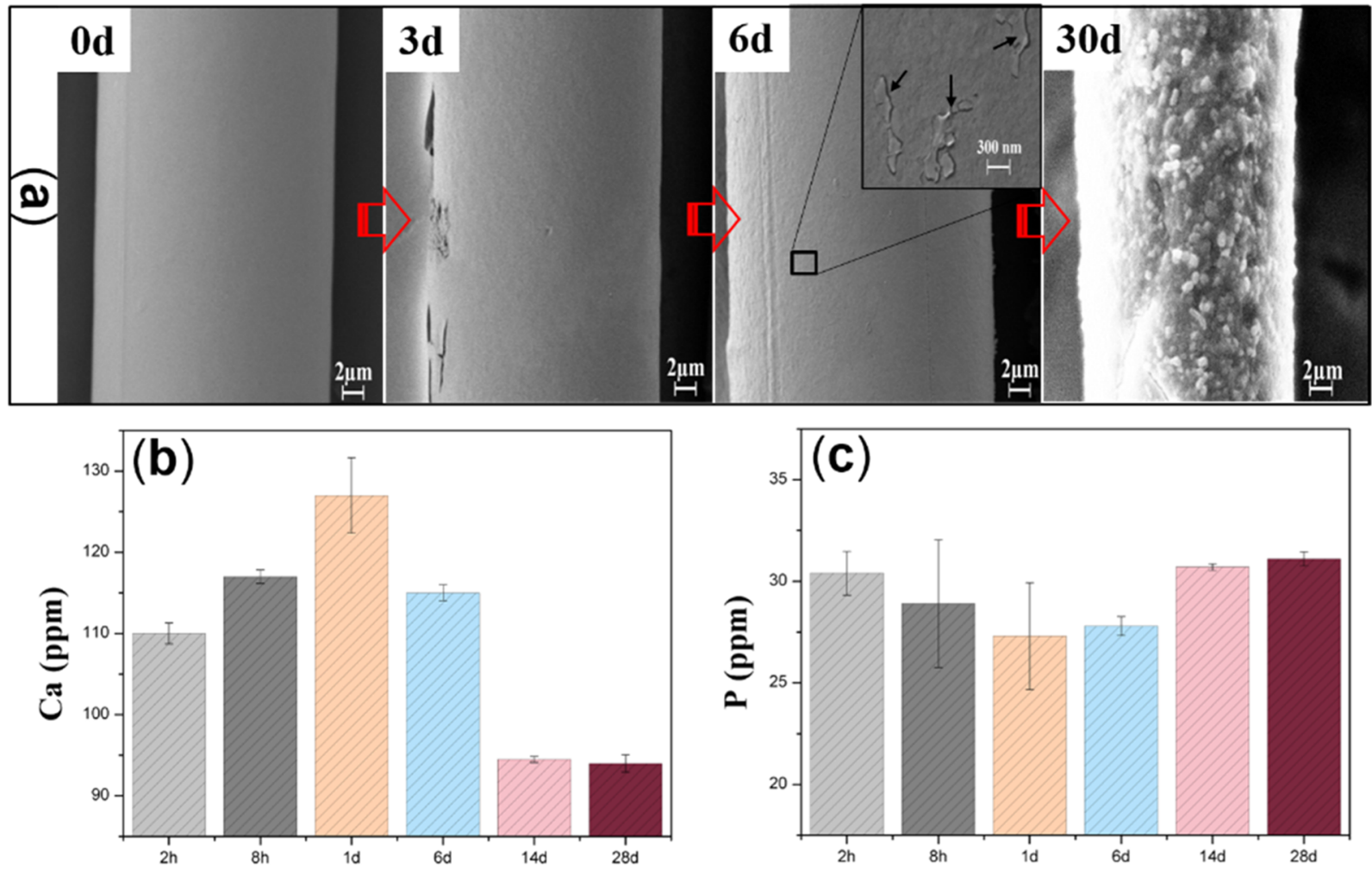

Figure 4. (a) Surface morphology of sPGF on the deposited sPGF-PAA coating as a function of incubation time in SBF. (b) Phosphorous and (c) calcium ion release profiles of sPGF in SBF $(0.1 \mathrm{~g} / 50 \mathrm{~mL})$ for up to 28 days.

312 according to the observations on samples III-VI, it was found 313 that the combination of high voltage with short deposition time 314 is also beneficial for achieving a high degree of alignment. The 315 effect of sPGF concentration on the degree of alignment was 316 investigated by comparative studies from two groups (samples I 317 vs III and samples VII vs VIII), which indicated a higher degree 318 of sPGF orientation obtained using a lower concentration of 319 sPGF.

320 The degree of sPGF alignment obtained using different EPD 321 parameters and SPGF concentrations was plotted in a 322 deposition voltage vs deposition time parameter window, as
PAA concentration and the deposition voltage play an 324 important role in the degree of orientation of sPGF. A high 325 concentration of PAA (charger) or sPGF will significantly 326 increase the deposit yield of randomly orientated SPGF on the 327 coating. Similarly, a higher deposition voltage will lead to 328 substantially higher driving force to accelerate the electro- 329 phoresis of SPGF in a short time. On the other hand, although 330 the electrophoresis of sPGF will be probably restricted under a 331 weak electric field, it is difficult to generate a relatively thick 332 PAA gel with an extremely low voltage or short deposition 333 time. In this way, if the deposition voltage is relatively low to 334 trigger preferentially the electrophoresis of PAA molecules 335 



Figure 5. (a) Cell viability cultured on sPGF-PAA and PAA (control) coatings for 2, 4, and 7 days. (b) SEM images of the cells cultured on sPGFPAA coatings for 2 days, and the arrows show the elongated skeleton and filaments along the fiber axis. Fluorescent images of stained cells on PAA (c) and sPGF-PAA (d) coatings for 2 days.

336 against the viscous suspension, a PAA-rich layer will be formed 337 during EPD. For example, in sample I (deposition voltage and 338 time of $10 \mathrm{~V}$ and $60 \mathrm{~s}$, respectively), a relative thick PAA-rich 339 layer was probably generated, resulting in a high degree of 340 sPGF alignment during the withdrawal process (rearrangement 341 of sPGF by fluid shear force). The PAA layer formed should be 342 thick enough to support the adhesion of sPGF during the 343 withdrawal process, e.g., the thickness of PAA layer on sample $344 \mathrm{VI}$ is only $\sim 3 \mu \mathrm{m}$ according to the cross-sectional SEM image 345 (Figure 3b), whereas sPGF were firmly adhered to the PAA 346 matrix even after mechanical cutting. The enhancement of PAA 347 deposition by increasing either deposition voltage or deposition 348 time will simultaneously deteriorate the degree of sPGF 349 alignment. Therefore, it is necessary to balance the deposition 350 of PAA layer and the degree of sPGF alignment by using proper 351 EPD parameters. In fact, there is a window rather than a point 352 of PAA thickness to obtain oriented sPGF structures according 353 to our experimental results. Based on this, there is another 354 possibility to yield a PAA-rich layer is the use of high deposition 355 voltage combined with short deposition time, as presented in 356 sample VI (deposition voltage and time of $50 \mathrm{~V}$ and $5 \mathrm{~s}$, 357 respectively). Under such EPD conditions, sufficient amount of 358 PAA gel can be generated, whereas few sPGF were able to 359 adhere to the electrode during the short deposition period. 360 Therefore, to ensure a high degree of alignment, a relatively 361 thick PAA layer should be predeposited while minimizing the deposition of sPGF during EPD, which could be realized by 362 applying a high voltage combined with a short time or a low 363 voltage combined with a long time, as indicated by the green 364 symbols in Figure 3.

3.2. In vitro Mineralization Study. The prepared sPGF- 366 PAA coating was incubated in SBF for up to 30 days to evaluate 367 their HA-forming ability as a marker of the coating 368 bioactivity. ${ }^{34}$ The surface morphology of sPGF as a function 369 of incubation time is shown in Figure 4a. The retained sPGF on $370 \mathrm{f} 4$ the coatings after SBF incubation also proves the high stability 371 and robust adhesion strength of SPGF-PAA coatings. The as- 372 deposited sPGF presented a smooth morphology and a 373 uniform diameter of $\sim 20 \mu \mathrm{m}$. The surface of sPGF became 374 rougher with longer incubation time, e.g., the fiber was 375 completely wrapped with a rough layer after 7 days of 376 incubation (shown in the inset for the day 7 sample). A slight 377 reduction in fiber diameter was also observed $(2-3 \mu \mathrm{m})$ after 378 30 days of incubation. Furthermore, the fiber was wrapped with 379 a cauliflower-like layer of spherical nanoparticles, which could 380 be assigned to the typical morphology of mineralized apatite 381 crystals in SBF. ${ }^{40,41}$

Ion release profiles of raw sPGF incubated in SBF were 383 analyzed to further elucidate the mineralization process of 384 sPGF (sPGF/SBF ratio of $0.1 \mathrm{~g} / 50 \mathrm{~mL}$ used in this 385 experiment). The dynamic concentrations of calcium and 386 phosphorous in the medium, which are closely associated with 387 

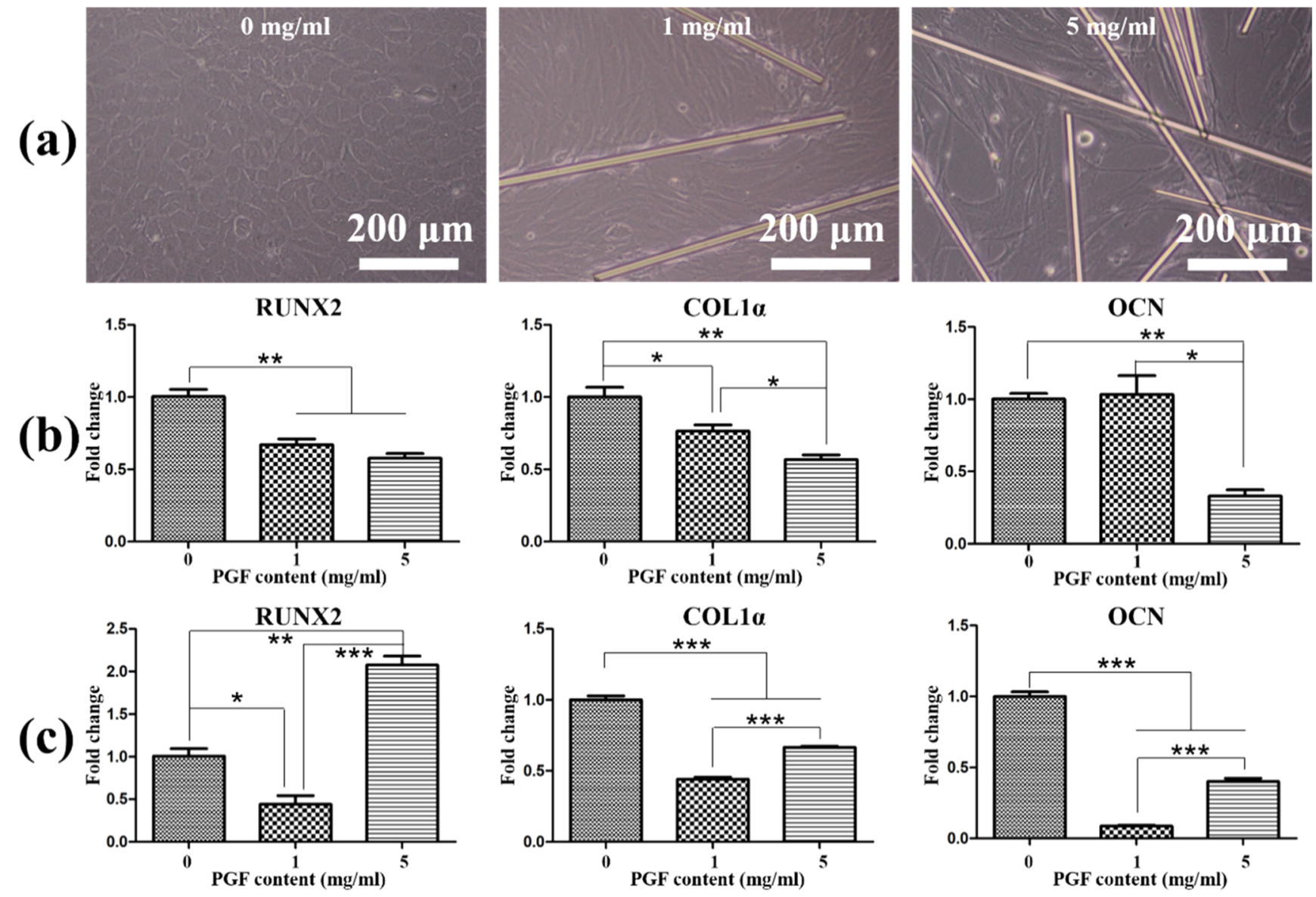

Figure 6. (a) Morphology of cells cultured with different concentrations of sPGF for 4 days. Osteogenesis-related gene expression of cells cultured with different concentrations of sPGF for (b) 7 days and (c) 14 days, respectively.

388 the formation of $\mathrm{HA}$ phase, are plotted in Figure $4 \mathrm{~b}, \mathrm{c}$, 389 respectively. According the SBF recipe provided by Kokubo et 390 al. $^{34}$ the initial concentrations of calcium and phosphorous in $391 \mathrm{SBF}$ are $2.5(100)$ and $1(31) \mathrm{mM}(\mathrm{ppm})$, respectively. The 392 ionic concentration of calcium and phosphorous should 393 increase as a result of the continuous degradation of sPGF. 394 However, the detected concentration of phosphorous exhibited 395 an appreciable reduction during the first 6 days of incubation, 396 whereas calcium exhibited a peak concentration of $\sim 127 \mathrm{ppm}$ 397 after 1 day of incubation and then decreased to $\sim 95 \mathrm{ppm}$ after 39814 days of incubation. It is reported that the degradation 399 products of sPGF, including $\mathrm{Ca}^{2+}$ and $\mathrm{PO}_{4}{ }^{3-}$ ions, will lead to 400 the supersaturation in the surrounding $\mathrm{SBF}$ to accelerate apatite 401 formation on the surface of the material. ${ }^{40}$ According to the 402 analyses of both the morphology and ion release profiles of 403 sPGF in SBF, it could be concluded that the formation of a 404 phosphate-rich layer was achieved at the early stage of SBF 405 incubation. In addition, the constant ionic concentration after 40614 days of incubation could be attributed to the equilibrium of 407 reactions involving the degradation of SPGF and formation of 408 apatite layers.

409 3.3. Cell Proliferation and Morphology on SPGF-PAA 410 Coatings. Cell viability on both PAA and sPGF-PAA coatings 411 was quantitatively studied by means of CCK-8 assay, as shown 412 in Figure 5a. Both samples exhibited comparable cell viability 413 after culture for 2 days, whereas a significantly higher cell 414 viability $(P<0.001)$ on the sPGF-PAA coatings was detected 415 after culture for 4 and 7 days. Both low- and high-magnification
SEM images of cell morphology on day 2 samples are shown in 416 Figure $5 \mathrm{~b}$. The cells tended to migrate to sPGF and spread 417 along the fiber axis, e.g., the length of a cell spreading on the 418 fiber is as high as $140 \mu \mathrm{m}$. In addition, it was observed that the 419 cells tightly adhered on the fiber surface and produced an 420 extremely elongated filopodia along the fiber axis. The obtained 421 results suggested that the presence of sPGF could promote 422 osteoblast proliferation. The bioactive composition of SPGF 423 provides favorable surfaces to induce cell attachment. More- 424 over, the high aspect ratio of sPGF provides spaces for cell 425 migration and proliferation. The cell morphology on PAA and 426 sPGF-PAA coatings in a relatively large area was also observed, 427 as shown in Figure $5 c, d$, respectively. The cells on the PAA 428 coatings presented a tabular morphology, whereas those on the 429 sPGF-PAA coatings presented an extremely elongated 430 morphology under the same incubation conditions. The 431 obtained results indicate that the sPGF will not only enhance 432 cell proliferation but also regulate cell migration and 433 morphology in vitro, e.g., accelerated oriented cell proliferation 434 in one dimension was obtained by culturing cells on the SPGF- 435 PAA coatings.

3.4. Osteogenic Differentiation Effect of sPGF. It is 437 likely that both the composition and fiber orientation of the 438 sPGF-PAA coating will influence the osteogenic activities. To 439 understand the specific effect of SPGF on osteogenic differ- 440 entiation while eliminating the possible interference induced by 441 fiber alignment, sPGF $(0,1$, and $5 \mathrm{mg} / \mathrm{mL})$ were homoge- 442 neously dispersed in the culture medium with MC3T3-E1 cells 443 
444 for a certain period. The selected concentrations of free sPGF 445 were determined based on the deposit yield of sPGF in the 446 coating (about $0.33-3.3 \mathrm{mg} / \mathrm{cm}^{2}$ ). The morphology of cells 447 cultured with different concentrations of sPGF for 4 days is 448 shown in Figure 6a. The cells cultured without sPGF achieved 449 complete confluence, whereas unconfluent areas increased with 450 increasing sPGF concentration. Moreover, compared to the 451 tabular geometry of the cells on the control sample, it was 452 observed that the cells cultured with sPGF exhibited an 453 elongated geometry along the fiber axis or between the fibers, 454 which is probably caused by stretching of the cell filopodia 455 during migration to sPGF and during proliferation along the 456 SPGF axes. The expression of osteogenesis-related genes of 457 MC3T3-E1 cell, including RUNX2, COL1 $\alpha$, and OCN, 458 cultured with different concentrations of sPGF for 7 and 14 459 days is shown in Figure $6 \mathrm{~b}, \mathrm{c}$, respectively. At day 7 , the 460 expression levels of all genes on sPGF-containing samples were 461 slightly lower than those on the control group (except OCN 462 expression with $1 \mathrm{mg} / \mathrm{mL}$ of sPGF), and the gene expression 463 became lower with increasing sPGF concentration. At day 14, 464 the relative gene expression was significantly increased with 465 increasing SPGF concentration; however, only the RUNX2 with $46610 \mathrm{mg} / \mathrm{mL}$ of sPGF exhibited a higher expression level 467 compared to the control group.

468 The ALP activity is considered a phenotypic marker for the 469 mature osteoblast, which is expected to be upregulated during 470 the early differentiation period, and gradually downregulated at 471 the later differentiation period. ${ }^{42,43}$ The ALP activity of cells 472 cultured with different concentrations of PGF for 7, 14, and 21 473 days is comparatively shown in Figure 7. After 7 days, the ALP

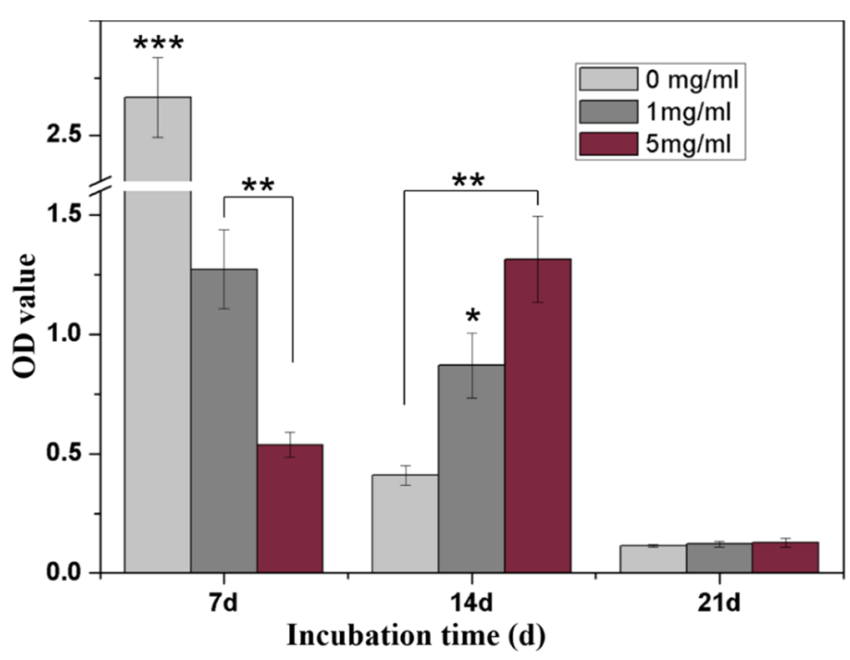

Figure 7. ALP activity of cells cultured with different concentrations of PGF. Error bars represent means \pm SD for $n=4\left({ }^{*} P<0.05\right.$, $* * P<$ $0.01, * * * P<0.001)$.

474 activity was significantly minimized with increasing sPGF 475 concentration. Moreover, the ALP activity was further reduced 476 on the sPGF-free sample and the sample with $1 \mathrm{mg} / \mathrm{mL}$ of 477 sPGF after culture for 14 days, whereas the sample with $5 \mathrm{mg}$ / $478 \mathrm{~mL}$ of sPGF exhibited increased and higher ALP activity than 479 the other two samples. After culture for 21 days, all samples 480 exhibited a substantially lowered ALP activity and no significant 481 difference was found according to the statistical analysis.

482 According to the previous CCK- 8 analysis, significantly 483 enhanced cell proliferation was detected when culturing with 484 sPGF. Meanwhile, the increase in the unconfluent area in
Figure 6a, probably due to preferential cell attachment on the 485 sPGF surfaces, indicated that a prolonged period of cell 486 proliferation is needed to cover the entire surface area. The 487 obtained results suggest that the delayed osteogenic differ- 488 entiation with increasing sPGF concentration at day 7 may be 489 attributed to the preferential cell proliferation against cell 490 differentiation. ${ }^{44,45}$ In addition, the surface topography of a 491 material also plays an important role in the osteogenic 492 functions by regulating the morphological characteristics of 493 the cell body and nucleus. ${ }^{46}$ It was reported that elongated 494 human mesenchymal stem cells (hMSCs), induced by micro- 495 grooved surfaces $(2.75 \mu \mathrm{m})$, exhibited lowered osteogenesis 496 compared to polygonal-like hMSCs cultured on flat surfaces, 497 which is positively correlated with the downexpression of 498 several cell morphological indexes, including integrin $\beta 1$ and N- 499 cadherin. ${ }^{47}$ Considering that sPGF used in this study possessed 500 a diameter comparable to the size of the reported micro- 501 grooves, it is considered that the elongation of MC3T3-E1 cells 502 induced by sPGF, with respect to the tabular morphology of 503 cells in the control group, should be another important reason 504 for their downexpression of osteogenic genes at day 7. After 505 culture for 14 days, the significant upexpression of osteogenic 506 marker genes with increasing SPGF concentration can be 507 ascribed to different reasons. On the one hand, the depletion of 508 unconfluent space may induce the transition from cell 509 proliferation to cell differentiation stage, and the higher cell 510 density with increasing sPGF concentration should contribute 511 to an increased gene expression level. On the other hand, the 512 formation of a phosphate-rich layer became increasingly 513 obvious at longer incubation times (Figure 4), and such a 514 favorable surface chemistry should lead to a positive influence 515 on the seen osteogenic activity. The long-term ALP activity 516 analysis also confirmed the delayed osteogenic activity with an 517 increasing sPGF concentration at day 7 . The further decreased 518 ALP activity of the control group was in accordance with the 519 expression trend during osteogenic differentiation. ${ }^{43}$ However, 520 the higher ALP activity on PGF-containing samples at day 14, 521 compared to the control group, indicated that the presence of 522 sPGF positively affects the osteogenesis at the later incubation 523 period. According to our findings from cell culture experiments, 524 significantly enhanced and directional cell proliferation was 525 obtained in the presence of sPGF, which is of great interest for 526 bone defect repair. Moreover, delayed but significantly 527 enhanced osteogenic differentiation was observed with the 528 increase in SPGF concentration. The long-term mineralization 529 of sPGF as well as the in vivo performance of the coatings will 530 be considered in future studies to assess the true potential of 531 the coatings in orthopedic applications. We expect that the 532 bioactive characteristic of sPGF will further facilitate osteogenic 533 activity at different periods of bone regeneration.

534

\section{CONCLUSIONS}

sPGF-PAA composite coatings with random and one-dimen- 535 sional orientation of SPGF were successfully fabricated on 536 stainless steel plates according to vertical and horizontal EPD, 537 respectively. A vertical electric field configuration can effectively 538 yield a SPGF-PAA composite coating with random orientation 539 of sPGF. By applying a horizontal electric field, a PAA gel layer 540 was first electrodeposited in situ on the anode, followed by the 541 fluid shear force-induced alignment of sPGF along the 542 withdrawal direction of the deposition electrode. Both the 543 EPD parameters and the SPGF concentration play important 544 roles in determining the degree of alignment during horizontal 545 
546 EPD. The prerequisite for achieving highly aligned sPGF 547 structures is the formation of a PAA-rich layer using a high 548 deposition and/or a short deposition time, which could then 549 assist the adhesion and arrangement of sPGF during the 550 withdrawal process. Continuous mineralization, characterized 551 by the formation of a phosphate-rich layer around sPGF was 552 achieved during incubation in SBF. In addition, the presence of 553 highly aligned SPGF in the composite coating not only 554 enhanced osteoblastic proliferation but also regulated cell 555 migration and spreading in one-dimensional orientation. 556 Although delayed osteogenic differentiation with increasing 557 sPGF concentration was detected in the initial stage, it is 558 expected that bioactive sPGF coatings with controllable 559 orientation are promising for directional bone defect repair. 560 The versatility of EPD technique to manipulate macroscopic 561 glass fibers to obtain oriented coatings was validated in this 562 study. Ongoing investigations have proven that similar fiber 563 structures could be obtained using different composition of 564 SPGF and even polymer fibers, and further investigations will 565 consider the effect of the oriented sPGF-PAA coatings on 566 bone bonding in vivo.

\section{AUTHOR INFORMATION}

\section{Corresponding Authors}

$569 *$ E-mail: lutinglixinxin@nwpu.edu.cn (T.L.L.).

570 *E-mail: aldo.boccaccini@ww.uni-erlangen.de (A.R.B.).

571 ORCID

572 Qiang Chen: 0000-0001-8829-0679

573 Xianhu Liu: 0000-0002-4975-3586

574 Aldo R. Boccaccini: 0000-0002-7377-2955

575 Notes

576 The authors declare no competing financial interest.

\section{ACKNOWLEDGMENTS}

578 We thank Dr. Xiao Lin (Key Laboratory for Space Bioscience 579 and Biotechnology, School of Life Sciences, Northwestern 580 Polytechnical University) for experimental support. Financial 581 supports for this work from the State Key Laboratory of 582 Solidification Processing in NWPU (SKLSP201715) and the 583 Fundamental Research Funds for the Central Universities (No. 584 G2016KY0103) are acknowledged.

\section{$585 \square$ REFERENCES}

586 (1) Ince, A.; Rupp, J.; Frommelt, L.; Katzer, A.; Gille, J.; Lohr, J. F. Is 587 "Aseptic" Loosening of the Prosthetic Cup After Total Hip 588 Replacement Due to Nonculturable Bacterial Pathogens in Patients 589 with Low-Grade Infection? Clin. Infect. Dis. 2004, 39, 1599-1603.

590 (2) Chen, Q. Z.; Thouas, G. A. Metallic Implant Biomaterials. Mater. 591 Sci. Eng., $R$ 2015, 87, 1-57.

592 (3) Hammond, P. T. Building Biomedical Materials Layer-by-Layer. 593 Mater. Today 2012, 15, 196-206.

594 (4) Goodman, S. B.; Yao, Z. Y.; Keeney, M.; Yang, F. The Future of 595 Biologic Coatings for Orthopaedic Implants. Biomaterials 2013, 34, 596 3174-3183.

597 (5) Song, J.; Chen, Q.; Zhang, Y.; Diba, M.; Kolwijck, E.; Shao, J. L.; 598 Jansen, J. A.; Yang, F.; Boccaccini, A. R.; Leeuwenburgh, S. C. G. 599 Electrophoretic Deposition of Chitosan Coatings Modified with 600 Gelatin Nanospheres to Tune the Release of Antibiotics. ACS Appl. 601 Mater. Interfaces 2016, 8, 13785-13792.

602 (6) Riool, M.; de Breij, A.; de Boer, L.; Kwakman, P. H. S.; 603 Cordfunke, R. A.; Cohen, O.; Malanovic, N.; Emanuel, N.; Lohner, K.; 604 Drijfhout, J. W.; Nibbering, P. H.; Zaat, S. A. J. Controlled Release of 605 LL-37-Derived Synthetic Antimicrobial and Anti-Biofilm Peptides 606 SAAP-145 and SAAP-276 Prevents Experimental Biomaterial-
Associated Staphylococcus aureus Infection. Adv. Funct. Mater. 2017, 607 27, No. 1606623.

(7) Hench, L. L. Bioceramics - from Concept to Clinic. J. Am. Ceram. 609 Soc. 1991, 74, 1487-1510.

(8) Geuli, O.; Metoki, N.; Eliaz, N.; Mandler, D. Electrochemically 611 Driven Hydroxyapatite Nanoparticles Coating of Medical Implants. 612 Adv. Funct. Mater. 2016, 26, 8003-8010.

(9) Chen, Q.; Garcia, R. P.; Munoz, J.; de Larraya, U. P.; Garmendia, 614 N.; Yao, Q. Q.; Boccaccini, A. R. Cellulose Nanocrystals-Bioactive 615 Glass Hybrid Coating as Bone Substitutes by Electrophoretic Co- 616 deposition: In Situ Control of Mineralization of Bioactive Glass and 617 Enhancement of Osteoblastic Performance. ACS Appl. Mater. Interfaces 618 2015, 7, 24715-24725. 619

(10) Pishbin, F.; Mourino, V.; Flor, S.; Kreppel, S.; Salih, V.; Ryan, 620 M. P.; Boccaccini, A. R. Electrophoretic Deposition of Gentamicin- 621 Loaded Bioactive Glass/Chitosan Composite Coatings for Orthopae- 622 dic Implants. ACS Appl. Mater. Interfaces 2014, 6, 8796-8806. 623 (11) Sharmin, N.; Rudd, C. D. Structure, Thermal Properties, 624 Dissolution Behaviour and Biomedical Applications of Phosphate 625 Glasses and Fibres: A Review. J. Mater. Sci. 2017, 52, 8733-8760. 626

(12) Ahmed, I.; Collins, C. A.; Lewis, M. P.; Olsen, I.; Knowles, J. C. 627 Processing, Characterisation and Biocompatibility of Iron-Phosphate 628 Glass Fibres for Tissue Engineering. Biomaterials 2004, 25, 3223- 629 3232.

(13) Vitale-Brovarone, C.; Novajra, G.; Lousteau, J.; Milanese, D.; 631 Raimondo, S.; Fornaro, M. Phosphate Glass Fibres and Their Role in 632 Neuronal Polarization and Axonal Growth Direction. Acta Biomater. 633 2012, 8, 1125-1136.

(14) Joo, N. Y.; Knowles, J. C.; Lee, G. S.; Kim, J. W.; Kim, H. W.; 635 Son, Y. J.; Hyun, J. K. Effects of Phosphate Glass Fiber-Collagen 636 Scaffolds on Functional Recovery of Completely Transected Rat 637 Spinal Cords. Acta Biomater. 2012, 8, 1802-1812.

(15) Kim, Y. P.; Lee, G. S.; Kim, J. W.; Kim, M. S.; Ahn, H. S.; Lim, J. 639 Y.; Kim, H. W.; Son, Y. J.; Knowles, J. C.; Hyun, J. K. Phosphate Glass 640 Fibres Promote Neurite Outgrowth and Early Regeneration in a 641 Peripheral Nerve Injury Model. J. Tissue Eng. Regener. Med. 2015, 9, 642 236-246.

(16) Jiang, X.; Cao, H. Q.; Shi, L. Y.; Ng, S. Y.; Stanton, L. W.; Chew, 644 S. Y. Nanofiber Topography and Sustained Biochemical Signaling 645 Enhance Human Mesenchymal Stem Cell Neural Commitment. Acta 646 Biomater. 2012, 8, 1290-1302.

(17) Li, Y.; Dai, X.; Bai, Y.; Liu, Y.; Wang, Y.; Liu, O.; Yan, F.; Tang, 648 Z.; Zhang, X.; Deng, X. Electroactive BaTiO3 Nanoparticle-Function- 649 alized Fibrous Scaffolds Enhance Osteogenic Differentiation of 650 Mesenchymal Stem Cells. Int. J. Nanomed. 2017, 12, 4007-4018. 651

(18) Reinwald, Y.; El Haj, A. J. Hydrostatic Pressure in Combination 652 with Topographical Cues Affects the Fate of Bone Marrow-Derived 653 Human Mesenchymal Stem Cells for Bone Tissue Regeneration. J. 654 Biomed. Mater. Res., Part A 2018, 106, 629-640.

(19) Rowland, D. C.; Aquilina, T.; Klein, A.; Hakimi, O.; Alexis- 656 Mouthuy, P.; Carr, A. J.; Snelling, S. J. A Comparative Evaluation of 657 the Effect of Polymer Chemistry and Fiber Orientation on 658 Mesenchymal Stem Cell Differentiation. J. Biomed. Mater. Res., Part 659 A 2016, 104, 2843-2853.

(20) Gao, X.; Song, J.; Zhang, Y.; Xu, X.; Zhang, S.; Ji, P.; Wei, S. 661 Bioinspired Design of Polycaprolactone Composite Nanofibers as 662 Artificial Bone Extracellular Matrix for Bone Regeneration Application. 663 ACS Appl. Mater. Interfaces 2016, 8, 27594-27610.

664

(21) Valente, T. A. M.; Silva, D. M.; Gomes, P. S.; Fernandes, M. H.; 665 Santos, J. D.; Sencadas, V. Effect of Sterilization Methods on 666 Electrospun Poly(lactic acid) (PLA) Fiber Alignment for Biomedical 667 Applications. ACS Appl. Mater. Interfaces 2016, 8, 3241-3249. 668

(22) Ma, J.; He, X. Z.; Jabbari, E. Osteogenic Differentiation of 669 Marrow Stromal Cells on Random and Aligned Electrospun Poly(L- 670 lactide) Nanofibers. Ann. Biomed. Eng. 2011, 39, 14-25. 671

(23) Kantawong, F.; Burgess, K. E. V.; Jayawardena, K.; Hart, A.; 672 Burchmore, R. J.; Gadegaard, N.; Oreffo, R. O. C.; Dalby, M. J. Whole 673 Proteome Analysis of Osteoprogenitor Differentiation Induced by 674 
675 Disordered Nanotopography and Mediated by ERK Signalling. 676 Biomaterials 2009, 30, 4723-4731.

677 (24) Wang, W.; Liu, Q.; Zhang, Y. M.; Zhao, L. Z. Involvement of $678 \mathrm{ILK} /$ ERK1/2 and ILK/p38 Pathways in Mediating the Enhanced 679 Osteoblast Differentiation by Micro/Nanotopography. Acta Biomater. 680 2014, 10, 3705-3715.

681 (25) Zhao, C.; Xia, L. G.; Zhai, D.; Zhang, N.; Liu, J. Q.; Fang, B.; 682 Chang, J.; Lin, K. L. Designing Ordered Micropatterned Hydrox683 yapatite Bioceramics to Promote the Growth and Osteogenic 684 Differentiation of Bone Marrow Stromal Cells. J. Mater. Chem. B 685 2015, 3, 968-976.

686 (26) Aminuddin, N. I.; Ahmad, R.; Akbar, S. A.; Pingguan-Murphy, 687 B. Osteoblast and Stem Cell Response to Nanoscale Topographies: A 688 Review. Sci. Technol. Adv. Mater. 2016, 17, 698-714.

689 (27) Abou Neel, E. A.; Pickup, D. M.; Valappil, S. P.; Newport, R. J.; 690 Knowles, J. C. Bioactive functional materials: a perspective on 691 phosphate-based glasses. J. Mater. Chem. 2009, 19, 690-701.

692 (28) Boccaccini, A. R; Keim, S.; Ma, R; Li, Y.; Zhitomirsky, I. 693 Electrophoretic Deposition of Biomaterials. J. R. Soc., Interface 2010, 7, 694 S581-S613.

695 (29) Yao, Q.; Jing, J. J.; Zeng, Q. Y.; Lu, T. L.; Liu, Y.; Zheng, X.; 696 Chen, Q. Bilayered BMP2 Eluting Coatings on Graphene Foam by 697 Electrophoretic Deposition: Electroresponsive BMP2 Release and 698 Enhancement of Osteogenic Differentiation. ACS Appl. Mater. 699 Interfaces 2017, 9, 39962-39970.

700 (30) Chen, Q.; de Larraya, U. P.; Garmendia, N.; Lasheras-Zubiate, 701 M.; Cordero-Arias, L.; Virtanen, S.; Soccaccini, A. R. Electrophoretic 702 Deposition of Cellulose Nanocrystals (CNs) and CNs/Alginate 703 Nanocomposite Coatings and Free Standing Membranes. Colloids 704 Surf., B 2014, 118, 41-48.

705 (31) Felfel, R. M.; Ahmed, I.; Parsons, A. J.; Walker, G. S.; Rudd, C. 706 D. In Vitro Degradation, Flexural, Compressive and Shear Properties 707 of Fully Bioresorbable Composite Rods. J. Mech. Behav. Biomed. Mater. 708 2011, 4, 1462-1472.

709 (32) Kato, N.; Schuetz, P.; Fery, A.; Caruso, F. Thin Multilayer Films 710 of Weak Polyelectrolytes on Colloid Particles. Macromolecules 2002, 711 35, 9780-9787.

712 (33) Wang, Y.; Deen, I.; Zhitomirsky, I. Electrophoretic Deposition 713 of Polyacrylic Acid and Composite Films Containing Nanotubes and 714 Oxide Particles. J. Colloid Interface Sci. 2011, 362, 367-374.

715 (34) Kokubo, T.; Takadama, H. How Useful is SBF in Predicting in 716 Vivo Bone Bioactivity? Biomaterials 2006, 27, 2907-2915.

717 (35) Cerruti, M.; Greenspan, D.; Powers, K. Effect of $\mathrm{pH}$ and Ionic 718 Strength on the Reactivity of Bioglass $((\mathrm{R}))$ 45S5. Biomaterials 2005, 719 26, 1665-1674.

720 (36) Hu, L.-f.; Li, J.-B.; Qian, A. R.; Wang, F.; Shang, P. 721 Mineralization Initiation of MC3T3-E1 Preosteoblast is Suppressed 722 Under Simulated Microgravity Condition. Cell Biol. Int. 2015, 39, 723 364-372.

724 (37) Stuart, M. A. C.; Huck, W. T. S.; Genzer, J.; Muller, M.; Ober, 725 C.; Stamm, M.; Sukhorukov, G. B.; Szleifer, I.; Tsukruk, V. V.; Urban, 726 M.; Winnik, F.; Zauscher, S.; Luzinov, I.; Minko, S. Emerging 727 Applications of Stimuli-Responsive Polymer Materials. Nat. Mater. 728 2010, 9, 101-113.

729 (38) Xiong, L.; Yang, T. S.; Yang, Y.; Xu, C. J.; Li, F. Y. Long-Term in 730 Vivo Biodistribution Imaging and Toxicity of Polyacrylic Acid-Coated 731 Upconversion Nanophosphors. Biomaterials 2010, 31, 7078-7085.

732 (39) Yoshioka, T.; Chavez-Valdez, A.; Roether, J. A.; Schubert, D. 733 W.; Boccaccini, A. R. AC Electrophoretic Deposition of Organic734 Inorganic Composite Coatings. J. Colloid Interface Sci. 2013, 392, 167735171.

736 (40) Massera, J.; Ahmed, I.; Petit, L.; Aallos, V.; Hupa, L. Phosphate737 Based Glass Fiber vs. Bulk Glass: Change in Fiber Optical Response to 738 Probe in Vitro Glass Reactivity. Mater. Sci. Eng., C 2014, 37, 251-257. 739 (41) Zheng, K.; Wu, Z. Y.; Wei, J.; Russel, C.; Liang, W.; Boccaccini, 740 A. R. Preparation and Characterization of Fibrous Chitosan-Glued 741 Phosphate Glass Fiber Scaffolds for Bone Regeneration. J. Mater. Sci.: 742 Mater. Med. 2015, 26, 224.
(42) Chen, Q.; Li, W.; Yao, Q. Q.; Liang, R. F.; Perez-Garcia, R.; 743 Munoz, J.; Boccaccini, A. R. Multilayered Drug Delivery Coatings 744 Composed of Daidzein-Loaded PHBV Microspheres Embedded in a 745 Biodegradable Polymer Matrix by Electrophoretic Deposition. J. Mater. 746 Chem. B 2016, 4, 5035-5045.

(43) Frith, J.; Genever, P. Transcriptional Control of Mesenchymal 748 Stem Cell Differentiation. Transfus. Med. Hemother. 2008, 35, 216- 749 227.

(44) Chang, B.; Song, W.; Han, T. X.; Yan, J.; Li, F. P.; Zhao, L. Z.; 751 Kou, H. C.; Zhang, Y. M. Influence of Pore Size of Porous Titanium 752 Fabricated by Vacuum Diffusion Bonding of Titanium Meshes on Cell 753 Penetration and Bone Ingrowth. Acta Biomater. 2016, 33, 311-321. 754

(45) Yan, J.; Zhang, C. C.; Zhao, Y. T.; Cao, C.; Wu, K. M.; Zhao, L. 755 Z.; Zhang, Y. M. Non-Viral Oligonucleotide AntimiR-138 Delivery to 756 Mesenchymal Stem Cell Sheets and the Effect on Osteogenesis. 757 Biomaterials 2014, 35, 7734-7749.

(46) Gong, T.; Xie, J.; Liao, J. F.; Zhang, T.; Lin, S. Y.; Lin, Y. F. 759 Nanomaterials and Bone Regeneration. Bone Res. 2015, 3, No. 15029. 760 (47) Kim, J.; Kim, H. N.; Lim, K. T.; Kim, Y.; Seonwoo, H.; Park, S. 761 H.; Lim, H. J.; Kim, D. H.; Suh, K. Y.; Choung, P. H.; Choung, Y. H.; 762 Chung, J. H. Designing Nanotopographical Density of Extracellular 763 Matrix for Controlled Morphology and Function of Human 764 Mesenchymal Stem Cells. Sci. Rep. 2013, 3, No. 3552. 